\title{
VANTAGEM COMPETITIVA NA GESTÃO SUSTENTÁVEL DA CADEIA DE SUPRIMENTOS: UM METAESTUDO
}

COMPETITIVE ADVANTAGE AND SUSTAINABLE SUPPLY CHAIN MANAGEMENT: A META-ANALISYS

VENTAJA COMPETITIVA EN LA GESTIÓN SOSTENIBLE DE LA CADENA DE SUMINISTRO: UN METAESTUDIO

\begin{abstract}
RESUMO
Questões vinculadas aos temas de responsabilidade social e de sustentabilidade vêm sendo cada vez mais recorrentes e representam desafios a serem trabalhados em diversas linhas de pesquisa e nas mais variadas formas de negócio. As abordagens vão do aspecto normativo à busca de desempenho superior e vantagem competitiva. Os desdobramentos na relação com os stakeholders são verificados na gestão da cadeia de suprimentos, foco de interesse do presente estudo. A metodologia é desenvolvida por meio de um metaestudo de artigos empíricos publicados. O objetivo é investigar se as estratégias socioambientais aplicadas à gestão da cadeia de suprimentos convergem para a gestão de stakeholders e o desenvolvimento de capacidades dinâmicas (dynamic capabilities) e, por fim, se configuram uma fonte de vantagem competitiva para as empresas investigadas.
\end{abstract}

PALAVRAS-CHAVE Vantagem competitiva, gestão sustentável da cadeia de suprimentos, cadeia verde de suprimentos, gestão de stakeholders, capacidades dinâmicas.

Renata Peregrino de Brito renatap.brito@gmail.com

Doutoranda em Administração de Empresas pela Escola de Administração de Empresas de São Paulo, Fundação Getulio Vargas - São Paulo - SP, Brasil

Patricia Calicchio Berardi patricia.berardi@terra.com.br

Doutoranda em Administração de Empresas pela Escola de Administração de Empresas de São Paulo, Fundação Getulio Vargas - São Paulo - SP, Brasil

Recebido em 28.05.2009. Aprovado em 23.04.2010

Avaliado pelo sistema double blind review. Editores Científicos: José Carlos Barbieri, Isabella Freitas Gouveia de Vasconcelos, Tales Andreassi e Flávio Carvalho de Vasconcelos

\begin{abstract}
Issues related to the themes of social responsibility and sustainability are increasingly recurrent and represent challenges to be addressed by various research lines and the most types of business. The approaches range from the normative aspect to the research for superior performance and competitive advantage. Implications of the relationship with stakeholders in supply chain management, which are the focus of this study, are verified. The employed methodology was that of a meta-study of empirical articles that have been published. The objective is to investigate whether the social environmental strategies applied to the supply chain converge for stakeholder management and the development of dynamic capabilities and, finally, whether they represent a source of competitive advantage for the companies investigated.

KEYWORDS Competitive advantage, sustainable supply chain management, green supply chain, stakeholder management, dynamic capabilities.

RESUMEN Las cuestiones relacionadas a los temas de responsabilidad social y sostenibilidad son cada vez más recurrentes y constituyen desafíos que deben ser trabajados en distintas líneas de investigación y en las más variadas formas de negocio. Los abordajes van desde el aspecto normativo a la búsqueda de desempeño superior y ventaja competitiva. Las repercusiones en la relación con los stakeholders son verificados en la gestión de la cadena de suministro, foco de interés del presente estudio. La metodología se desarrolla a través de un metaestudio de artículos empíricos publicados. El objetivo es investigar si las estrategias socioambientales aplicadas a la gestión de la cadena de suministro convergen con la gestión de stakeholders y el desarrollo de capacidades dinámicas (dynamic capabilities) y, finalmente, si configuran una fuente de ventaja competitiva para las empresas investigadas.
\end{abstract}

PALABRAS CLAVE Ventaja competitiva, gestión sostenible de la cadena de suministro, cadena verde de suministro, gestión de stakeholders, capacidades dinámicas. 


\section{INTRODUÇÃO}

O presente trabalho está dividido em cinco seções. $\mathrm{Na}$ primeira, é feita uma revisão da temática de responsabilidade socioambiental no campo da Administração, chegando ao aspecto mais estratégico da gestão de recursos naturais e da capacidade da relação com os stakeholders de levar a uma vantagem competitiva para a empresa. Na segunda seção, verifica-se a evolução dos temas socioambientais na relação com os parceiros da cadeia de suprimentos e quais são os tipos de definições trazidas para uma tentativa de inserir a sustentabilidade nessas relações. A descrição da metodologia cabe à terceira seção. A etapa seguinte apresenta a pesquisa de como os aspectos teóricos levantados até então são explorados em trabalhos empíricos, os resultados obtidos e elucida as principais indagações que motivaram o presente estudo. Ao final, na quinta e última seção, faz-se o fechamento do trabalho com as considerações finais e possibilidades para novas pesquisas.

A busca do entendimento do papel das empresas e suas respectivas responsabilidades é recorrente, tanto na discussão acadêmica em diferentes disciplinas de Direito, Economia, Administração, Sociologia, Antropologia, Filosofia, entre outras, como no âmbito gerencial nas diversas abordagens de gestão prática. Perspectivas sociais e questões ambientais passaram a ser introduzidas com maior frequência no debate econômico, o que conferiu uma gama variada de correntes, conceitos e definições, sendo notória a falta de consenso, sobretudo nos temas de responsabilidade social empresarial (COCHRAN e WOOD, 1984; MCGUIRE, SUNDGREN, SCHNEEWEIS, 1988; PRESTON e O'BANNON, 1997; VERSCHOOR, 1998; GERDE E WOKUTCH, 1998; ROWLEY E BERMAN, 2000; MARREWIJK, 2003; GARRIGA e MELÉ, 2004; BAKKER, GROENEWEGEN e HOND, 2005) e de sustentabilidade (LÉLÉ, 1991; BARONI, 1992; GOODLAND, 1995; MARREWIJK, 2003; FABER, JORNA, ENGELEN, 2005). Ainda assim, persiste o interesse em verificar possíveis convergências desses temas nas atividades gerenciais, suas influências e os impactos causados na estratégia de negócios.

O objetivo do estudo que ora se apresenta é, a partir da discussão da responsabilidade socioambiental das empresas que passaram a interiorizar em seus modelos de gestão a temática socioambiental, identificar sua evolução na formulação da estratégia empresarial. Mais especificamente, busca-se com a revisão de estudos empíricos recentemente publicados, perceber se há convergência entre propostas teóricas e as práticas pesquisadas.

\section{ESTRATÉGIA EMPRESARIAL E A AGENDA SOCIOAMBIENTAL}

A evolução da agenda socioambiental na formulação da estratégia empresarial começa a ser mais estudada na década de 1990. Estudos exploraram práticas socioambientais como fontes de vantagem competitiva por meio da legitimação social (SHRIVASTAVA, 1995b; WESTLEY e VREDENBURG, 1991), da redução de obrigações e custos para legalização (SHRIVASTAVA, 1995b) e do desenvolvimento de "melhores práticas" de negócios (SHRIVASTAVA, 1995; PORTER e VAN DER LINDE, 1995).

Práticas ambientais poderiam ser geradoras de vantagem competitiva por meio de estratégias de baixo custo ou diferenciação conforme as dimensões de competitividade estudadas por Porter (1980). A administração de recursos naturais de forma eficiente e a diminuição de perdas no processo produtivo são fontes de diminuição de custos. Essas práticas também poderiam levar empresas a alcançar uma diferenciação no contexto internacional, onde as restrições ambientais eram crescentes. A pressão regulatória também seria um estímulo à inovação nas empresas, superando a inércia e incentivando a criatividade nas organizações (PORTER e VAN DER LINDE, 1995).

A vantagem em custos é ainda endossada em iniciativas de processos aplicados, como ecocentric management (SHRIVASTAVA, 1995a) e design for disassembly (SHRIVASTAVA, 1995b). A proposta de gestão ecocêntrica (ecocentric management) faz uma contraposição ao modelo tradicional, buscando alinhar a gestão empresarial com a gestão de impactos no meio ambiente, sendo uma das consequências a redução na utilização de insumos (SHRIVASTAVA, 1995a). Fruto desse processo, o design for disassembly pressupõe o estudo do processo produtivo, propondo produtos que sejam fáceis de desmontar e reciclar (SHRIVASTAVA, 1995b). O resultado é o desenvolvimento de processos e produtos proprietários. A trajetória de construção desses processos e produtos pode tornálos ativos valiosos para a empresa (DIERICKX e COOL, 1989). O conceito de processo proprietário alinha-se com a lógica da visão baseada em recursos, Resource Based View (RBV), que foca os recursos idiossincráticos da empresa como fonte de vantagem competitiva (BARNEY, 1991).

Hart (1995) aplica, então, a RBV no estudo das estratégias ambientais e desenvolve a Natural-Resource-Based View. Segundo a Natural-Resource-Based View (HART, 1995), os desafios mundiais de escassez de recursos naturais serão inevitáveis limitadores da atividade econômica. A capacidade da empresa em lidar com tais restrições leva a um modelo que vai da prevenção à poluição ao 
desenvolvimento sustentável, passando pelo meticuloso processo de conhecimento de toda a cadeia do produto, o product stewarship.

As argumentações iniciais pelo "green is gold" são paulatinamente substituídas por argumentos baseados em recursos e na capacidade da empresa em explorar esses desafios (SHARMA e VREDENBURG, 1998). Vários estudos empíricos seguem a iniciativa de Hart (1995) incluindo a RBV como forma de avaliação da estratégia ambiental (RUSSO e FOUTS, 1997; SHARMA e VREDENBURG, 1998; CHRISTMANN, 2000). As conclusões não desmontam as argumentações de estratégias ambientais como um todo, mas também não concluem pela vantagem competitiva no acesso aos recursos naturais escassos ou pela liderança em processos (HART, 1995). Por outro lado, os estudos reforçam a importância da capacidade da integração dos stakeholders como fontes de inovação e aprendizado, por meio da triangulação de informações ambientais (SHARMA e VREDENBURG, 1998).

Buysse e Verbeke (2003) pesquisam empresas poluidoras da Bélgica na busca de evidências da ligação entre a gestão de stakeholders e estratégias ambientais. Os autores concluem que a gestão de stakeholders está relacionada ao desenvolvimento de competências, green competences (BUYSSE e VERBEKE, 2003); e que a liderança em práticas ambientais não estava relacionada à crescente importância da legislação ambiental, mas a atitudes voluntárias das empresas.

A discussão de estratégias ambientais passa a explorar a geração de capacidades nas organizações. As capacidades dinâmicas, dynamic capabilities, conferem habilidade na renovação de competências de acordo com as mudanças no ambiente e na busca de soluções inovadoras, garantido a competitividade da empresa (TEECE, PISANO, SHUEN, 1997; TEECE, 2007). O engajamento com os stakeholders e a busca de soluções permitiria à empresa desenvolver suas competências dinâmicas, que por fim se manifestam nos produtos e serviços (TEECE, PISANO, SHUEN, 1997).

Contudo, o debate de estratégias ambientais distanciase um pouco das argumentações de lideranças de custos, ecoeficiência ou produtos específicos. Mesmo reconhecendo essas estratégias como formas de competitividade (ORSATO, 2006), a prova pela vantagem não é tão clara ou óbvia. Dentro do conceito de "melhores práticas", várias estratégias ambientais podem ser imitadas e trariam então apenas paridade competitiva às empresas.

A pressão normativa e legal para a solução de problemas sociais e ambientais leva empresas a adotarem práticas so- cioambientais para a manutenção da licença de operação das empresas. A busca de melhores práticas e o enquadramento em padrões de operação resultam em um movimento isomórfico (DIMAGGIO e POWELL, 1983) e sem diferenciação. Alguns autores exploram, então, essa combinação de estratégia motivada tanto pela visão baseada em recursos como pelas pressões institucionais (BANSAL, 2005; DARNALL e EDWARDS JR, 2006), uma vez que tanto a pressão por padrões mínimos como a pressão por competitividade fazem parte da realidade das empresas.

Mas o estudo da formação de estratégias na interação com stakeholders parece o mais promissor. O tratamento dado às demandas e a configuração das respostas elaboradas na interação com stakeholders primários ou secundários demonstram a insurgência de atitudes socioambientais avançadas (SHARMA e HENRIQUES, 2005; EESLEY e LENOX, 2006).

Esse movimento é certamente parte da evolução, tanto na sua perspectiva como no foco, do debate de estratégia empresarial. As teorias geradas pela escola da organização industrial exploraram as diferenças entre indústrias e as economias de escala; a teoria de recursos idiossincráticos explora a organização e as economias de escala e de escopo. A crescente complexidade do ambiente passa a impor a necessidade do desenvolvimento de capacidades dinâmicas por meio do portfólio de relacionamentos (VENKATRAMAN e SUBRAMANIAM, 2002).

Sob a ótica do portfólio de relacionamentos, as empresas deixam de ser as unidades de análise, e as interações organizacionais passam a ser geradoras de valor e competitividade. $\mathrm{O}$ valor gerado no contexto entre organizações é também pesquisado em artigos empíricos (DYER, 1996; DYER e HATCH, 2006) e em uma abordagem relacional, proposta pela teoria do Relational View (DYER e SINGH, 1998).

O desenvolvimento da capacidade colaborativa, o aprendizado e a inovação gerados nas interações com stakeholders podem compor uma competência diferenciadora da empresa. Essa competência, na gestão das relações e nas trocas de conhecimento, pode ser aplicada mais amplamente pela empresa e vir a gerar rendas relacionais.

Por essa visão, a organização passa de uma lógica de gestão de contratos de curto prazo (WILLIAMSON, 1975) para uma lógica de potencial de geração de opções reais (VENKATRAMAN e SUBRAMANIAM, 2002). Ficam os desafios de lidar com o oportunismo e a capacidade de extração de valor dessas relações; esses tópicos são pesquisados nas interações da empresa com seus fornecedores, na literatura de gestão da cadeia de suprimentos. 


\section{GESTÃO DA CADEIA DE SUPRIMENTOS}

Verifica-se como evolução dos estudos em Gestão Operacional, tendo como base o veículo pertinente à área: Production and Operations Management, um enfoque de preocupação com eficiência e qualidade nos movimentos com ênfase na Gestão da Qualidade Total - Total Quality Management (TQM), e na sequência o direcionamento dos estudos recai em operações em tempo real - just in time (JIT), ao final da década de 1980. No início da década seguinte, o foco estava nos processos de reengenharia, com destaque para a criação de cadeia de valor, outsourcing e inovação ao longo da cadeia de suprimentos. Nesse período, passou-se a dar uma maior importância ao alinhamento entre questões estratégicas com questões operacionais. Com a nova dinâmica econômica de globalização de mercados, os gestores passaram a entender que a lucratividade não representava mais o único elemento de sucesso para longo prazo. Preocupações mais amplas com pessoas e o meio ambiente passaram a representar variáveis relevantes no processo operacional (KLEINDORFER, SINGHAL, VAN WASSENHOVE, 2005).

Para entender o processo de uma abordagem de cadeia de suprimento sustentável, faz-se necessária a compreensão de como as definições e conceitos vêm sendo trabalhados ao longo das últimas décadas no que concerne à cadeia produtiva e sua gestão.

Segundo Sampaio (2007), existem diferentes visões de Supply Chain Management (SCM), expressão que, traduzida, significa "gestão da cadeia de suprimentos", podendo ser logística, o que efetivamente não contemplaria uma abordagem integrada das diversas funções da cadeia (produção, suprimentos, compras, logística e relacionamentos com fornecedores e clientes). Num entendimento um pouco mais avançado, a SCM seria uma combinação de funções de suprimentos, produção e logística, abrangendo tarefas mais complexas. Um entendimento tanto quanto reducionista seria atribuir questões de tecnologia da informação como suficientes para lidar com o fluxo de informações da cadeia. Outra consideração abarcaria o abastecimento global de cadeias, contemplando a logística internacional com necessidade de ênfase em gestão de riscos, mas que ainda assim não representaria todo o universo associado à SCM. A definição de SCM como uma visão integrada de negócios parece ser a orientação que melhor contempla processos e gestão de informação de modo a agregar valor aos clientes e demais stakeholders.

Para Andersen e Skjoett-Larsen (2009), a gestão da cadeia de suprimentos representa uma área de forte importância estratégica por aspectos como competição global, outsourcing de atividades não centrais, pequeno ciclo de vida de produtos e compressão de tempo em diferentes etapas da cadeia. A capacidade em estabelecer um estreito e duradouro relacionamento com parceiros estratégicos tornou-se fator crucial para criar vantagem competitiva.

A SCM passou de perspectivas operacionais (compras, qualidade e outros) para perspectivas estratégicas com foco na relação de longo prazo com parceiros estratégicos (ANDERSEN e SKJOETT-LARSEN, 2009).

\section{Gestão sustentável da cadeia de suprimentos}

Com uma crescente demanda em integrar questões ambientais e sociais à gestão da cadeia de suprimentos por meio de pressões externas aos negócios foi possível perceber o surgimento de uma aproximação de conceitos utilizados no tema da sustentabilidade com a gestão operacional da cadeia de suprimentos: tanto pelo lado da preocupação com os recursos, que podem ser entendidos como finitos, como ainda pela forma de processamento dos produtos e serviços (KLEINDORFER, SINGHAL E VAN WASSENHOVE, 2005; LINTON, KLASSEN E JAYARAMAN, 2007; MARKLEY e DAVIS, 2007; ANDERSEN e SKJOETT-LARSEN, 2009).

Inicialmente, a gestão da cadeia de suprimentos focava, tanto em teoria quanto na prática, questões relacionadas à integração de processos entre parceiros da cadeia, análise de custo-eficiência dos fornecedores da cadeia e serviços aos consumidores. Contudo, com a elevação da discussão ambiental e social associada aos questionamentos sobre impactos de produção e consumo, novos interesses despontaram: logística reversa, gestão ambiental, cadeia de suprimento verde e cadeia de suprimento sustentável. Assim, percebe-se que a gestão sustentável de operações aproximou a visão tradicional de gestão de operações - lucro e eficiência, com aspectos mais amplos de impactos aos públicos de interesse e ao meio ambiente (KLEINDORFER, SINGHAL, VAN WASSENHOVE, 2005). Pode-se dizer, ainda, que a gestão sustentável da cadeia de suprimentos conduziu a uma expansão das fronteiras e passou a contemplar mais processos que os anteriormente inseridos na gestão da cadeia de suprimentos (SVENSSON, 2007).

Apesar do crescente interesse, nota-se certa diversidade de classificações e enquadramentos de trabalhos e pesquisas, tanto pela mídia, pela academia como também pelo mundo corporativo em que os temas tratados entre cadeia de suprimentos e questões ambientais e sociais podem aparecer em cadeia de suprimentos sustentável, gestão ambiental, triple bottom line, empresas verdes, fornecimento verde e responsabilidade social corporativa (ANDERSEN 
e SKJOETT-LARSEN, 2009). Adicionalmente, temas como estratégias de compras verdes, compras ambientais, marketing verde, produtos com diferenciação ambiental, logística reversa, rotulagem sustentável, ciclo de vida do produto, retorno de produtos, reciclagem, substituição de materiais, reuso de materiais, disposição final, recondicionamento e remanufatura são exemplos da vasta gama de possibilidades para abordagens de operações com sustentabilidade (SVENSSON, 2007).

Srivastava (2007) fez uma revisão de literatura a fim de levantar os diferentes entendimentos envolvidos na denominada gestão verde da cadeia de suprimentos. A verificação contemplou 1.500 trabalhos entre livros, artigos e publicações em língua inglesa com ênfase em veículos europeus e americanos, a partir de 1990, que, segundo o autor, parece ter sido o início dos debates sobre o tema. Feitos alguns filtros, a base de análise foi reduzida para 227 trabalhos. Foram definidas três classificações: literatura sobre importância de gestão verde da cadeia de suprimentos, literatura sobre design de produtos verdes e literatura sobre operações verdes. A constatação desse estudo enfatiza trabalhos quantitativos em diversas áreas, como: operações, estratégia, gestão da cadeia de suprimentos, tecnologia de produtos e processos. A análise de estudos empíricos demonstrou, entre outras coisas, que ainda existe necessidade em aprofundar os conhecimentos das áreas organizacionais comprometidas com a gestão verde da cadeia de suprimentos com um alinhamento das prioridades competitivas.

Para Rao e Holt (2005), a gestão verde da cadeia de suprimentos promove eficiência e sinergia entre os parceiros do negócio e contribui para uma aumento da performance ambiental, minimizando desperdícios e auxiliando a economia de custos. Sobre tal sinergia, segundo os autores, "espera-se um aumento de imagem corporativa, vantagem competitiva e exposição de marketing" (RAO e HOLT, 2005, p. 899, tradução nossa). Contudo, as empresas só adotam práticas de gestão verde da cadeia de suprimentos se identificarem benefícios, especificamente nos resultados financeiros e operacionais.

São apontadas como argumentação positiva para a gestão verde da cadeia de suprimentos a redução de custos operacionais, a integração de fornecedores no processo de tomada de decisão, estratégias de compras diferenciadas, redução dos desperdícios, substituição de materiais e matérias-primas, redução das emissões de gases de efeito estufa, melhor utilização de recursos naturais, desenvolvimento mais eficiente de novos produtos, inovação, entre outros (RAO e HOLT, 2005).

Markley e Davis (2007) indagam como as empresas poderiam crescer e se diferenciar a partir de certo nivelamento ocorrido no mercado, quando as empresas competidoras passaram a acessar matérias-primas e fornecedores similares, limitando assim as habilidades organizacionais ao preço ou ao produto. São muitas as demandas externas: consumidores são direcionados por baixos preços, tecnologias avançadas, além dos governos que demandam, via regulamentação e políticas públicas, interação com meios externos às organizações. Uma possibilidade apontada pelas autoras está no desenvolvimento de uma organização sustentável - aquela que contribui para o desenvolvimento sustentável por meio da realização de suas atividades pelas premissas do triple bottom line, mais propriamente na sustentabilidade da cadeia de suprimentos.

$\mathrm{Na}$ revisão de literatura feita por Seuring e Müller (2008) após analisarem 191 artigos sobre gestão da cadeia de suprimentos sustentável no período de 1994 a 2007, tendo como foco três categorias: ambiental, social e ambas, em que buscavam identificar as diferentes pressões ou motivadores para a sustentabilidade na cadeia de suprimentos, os autores constataram como questões norteadoras dos trabalhos duas estratégias, o enfoque na performance econômica ou no desejo de buscar um produto sustentável.

Nota-se que, num primeiro momento, o interesse das pesquisas sobre sustentabilidade recaía em impactos financeiros causados por comportamentos ambientais específicos, tais como controle da poluição, reciclagem ou logística reversa. Poucos trabalhos abordaram o impacto da cadeia de suprimentos sustentável com foco na proteção global, ambiental e de capital social. Destaca-se a ausência de trabalhos sobre o potencial de desenvolvimento de vantagem competitiva entendido como base de uma cadeia de suprimentos sustentável (MARKLEY e DAVIS, 2007).

"A criação de uma cadeia de suprimentos sustentável pode constituir-se numa estratégia efetiva na busca de vantagem competitiva e assegurar aprovação futura dos stakeholders" (MARKLEY e DAVIS, 2007, p. 765, tradução nossa).

Percebe-se que, em certa quantidade de estudos realizados pelo enfoque da cadeia de suprimentos com aspectos socioambientais, a transição de perspectivas puramente operacionais para perspectivas estratégicas fez-se presente com destaque para a alegação de que gestões sustentáveis da cadeia trariam diferenciais nos desempenho e maior vantagem competitiva (KLEINDORFER, SINGHAL, VAN WASSENHOVE, 2005; RAO e HOLT, 2005; LINTON, KLASSEN, JAYARAMAN, 2007; MARKLEY E DAVIS, 2007; ANDERSEN e SKJOETT-LARSEN, 2009). Embora, 
em alguns casos, os autores atestem haver maior necessidade de aprofundar estudos sobre as vantagens competitivas advindas das gestões sustentáveis nas cadeias de suprimentos.

O estudo da Gestão Sustentável da Cadeia de Suprimentos - Sustainable Supply Chain Management (SSCM) parece seguir a linha do debate da agenda socioambiental na formulação da estratégia empresarial. Nesse sentido, são identificados como principais motores a necessidade de adequação às pressões ambientais e a busca de ecoeficiência, ou menores custos.

Resta saber se é possível reconhecer a SSCM e a relação com parceiros como fonte de vantagem competitiva, que seria a derivação esperada, porém ainda não pesquisada, daí o foco deste artigo.

\section{METODOLOGIA}

O principal interesse deste estudo é analisar a abordagem estratégica da linha de operações, mais precisamente nos trabalhos de cunho social e/ou ambiental, pela evolução da gestão sustentável da cadeia de suprimentos. A técnica utilizada é, portanto, um metaestudo dos artigos empíricos de periódicos constantes na revisão recente da literatura de SSCM realizada por Seuring e Müller (2008).

As técnicas de metaestudo são baseadas na sistematização de um conjunto de dados obtidos a partir da literatura científica. O levantamento da base de dados é fruto de uma organização lógica das informações descritas no material bibliográfico identificado previamente. A primeira fase de um trabalho pela abordagem meta-analítica é a codificação dos dados das publicações incluídas na base estabelecida. A análise gráfica de dados representa outra importante etapa de uma meta-análise. Essa análise permite identificar as informações e relações importantes definidas no objeto de pesquisa. O uso dessa técnica de análise apresenta certa recorrência nos trabalhos brasileiros nas áreas de Marketing, Administração da Informação, Produção, Administração Pública e Organizações, e parece ser interessante fonte de pesquisa para novos trabalhos (TONELLI e outros, 2003).

O termo "meta-análise", segundo Hedges (1992), foi introduzido em 1976 por Gene Glass como sendo "análise de resultados de análises estatísticas tendo como proposta o desenho de conclusões gerais" (HEDGES, 1992, p. 279 , tradução nossa). Desde a introdução do termo, o uso de métodos de análise que combinem resultados de estudos já realizados passou a ser amplamente utilizado em estudos de educação, psicologia e ciências biomédicas. $\mathrm{Na}$ literatura, encontra-se ainda como denominação para a técnica de investigação por meio de análise em resultados de pesquisas já realizadas em determinados temas, metaevolução ou metaestudo (PATTON, 2002). A meta-análise não preconiza o uso de uma técnica específica, qualitativa ou quantitativa, mas dentro da tradição de estudos da área de Administração acabou por estabelecer um padrão quantitativo de análise de artigos. A base de dados aqui pesquisada não permitia o uso de técnicas quantitativas, daí a denominação "metaestudo".

Tomou-se por base o artigo de Seuring e Müller (2008), em que foram analisados 191 artigos pertencentes a diversas bases. Por ser um levantamento recente sobre gestão sustentável da cadeia de suprimentos, e tendo sua estrutura contemplado publicações acadêmicas de material produzido em inglês com foco em gestão, ficaram eliminados, assim, trabalhos em outros idiomas e de cunho em ciências políticas ou puramente técnicos. Entendeu-se apropriada a utilização desse trabalho para a realização de um metaestudo. Ademais, um fator verificado na própria revisão de literatura de gestão sustentável na cadeia de suprimentos foi o destaque dado por diversos autores à ausência e à necessidade de maior produção sobre vantagem competitiva, como já apresentado na primeira parte deste estudo. Na contagem de palavras apresentada pelo artigo de Seuring e Müller (2008), "vantagem competitiva" aparece em 71 artigos ( $37 \%$ do total), porém sem maiores apreciações sobre a forma de abordagem do tema. Sendo assim, o estudo segue na busca de um entendimento da forma como a vantagem competitiva está inserida nas análises e resultados dos artigos levantados.

\section{PESQUISA}

Do total de 191 artigos identificados que compõem a base de dados do referido artigo, os autores a desmembraram na classificação de cinco tipos epistemológicos, a saber: teórico, modelo, revisão, estudo de caso e survey. Como a proposta deste estudo é analisar a abordagem empírica, o primeiro recorte elimina os artigos teóricos, de modelagem ou de revisão de literatura. Restringe-se então ao universo de 68 estudos de caso e mais 51 surveys, totalizando uma amostra selecionada de 119 artigos. Verificadas as restrições de acesso, 10 artigos foram retirados, totalizando 109 artigos, distribuídos em 38 diferentes veículos de publicação (journals), com destaque para o Greener Management International (18 artigos) e o Journal of Cleaner Production (13 artigos). A Tabela 1 apresenta a distribuição da concentração das maiores incidências. 
O metaestudo é conduzido por meio da revisão de 109 artigos empíricos, levantados na revisão de literatura conduzida por Seuring e Müller (2008) sobre SSCM no período de 1994 a 2007. Iniciada a primeira categorização, por tipo ou formato, apresentada quando da revisão dos resumos (abstracts), definiram-se quatro tipologias que demonstram a forma de tratamento da questão socioambiental: ética (0), adaptação (1), ecoeficiência (2) e estratégica (3). A análise buscou primeiramente classificar os estudos de acordo com a forma de inserção dos aspectos socioambientais na gestão da cadeia. O enfoque dos estudos é variado, podendo ser categorizado nos seguintes conceitos:

- Cat. 0 - Objetivos éticos, em que a importância é dada, nas relações sociais, na ética das tratativas. Por não abordarem a gestão de forma estratégica competitiva, os 12 artigos desta categoria foram excluídos.

- Cat. 1 - A necessidade de adaptação por pressão regulatória ou do ambiente competitivo. Apareceu como tópico em 28 artigos.

- Cat. 2 - A busca da ecoeficiência, com resultados em desempenho econômico e/ou ambiental, foi tema de 46 artigos.

- Cat. 3 - O enfoque estratégico apareceu na busca de parceiras para inovação, desenvolvimento de capacidades e vantagem competitiva. São 23 artigos nesta categoria

Segmentada por quartis (Gráfico 1), a produção distribuída nas categorias ao longo de 13 anos não tem forte

Tabela 1 - Representatividade por periódico

\begin{tabular}{|l|c|c|}
\hline \multicolumn{1}{|c|}{ PERIÓDICO } & TOTAL PUBLICAÇÕES & PORCENTUAL \% \\
\hline Business Strategy and the Enviroment & 7 & $6 \%$ \\
\hline Greener Management International & 18 & $15 \%$ \\
\hline International Journal of Operations \& Production Management & 7 & $6 \%$ \\
\hline Journal of Business Ethics & 5 & $4 \%$ \\
\hline Journal of Cleaner Production & 13 & $11 \%$ \\
\hline Journal of Operations Management & 5 & $4 \%$ \\
\hline Production and Operations Management & 5 & $4 \%$ \\
\hline Demais & 59 & $50 \%$ \\
\hline Total & 119 & 100 \\
\hline
\end{tabular}

\section{Gráfico 1 - Distribuição das categorias nos anos}

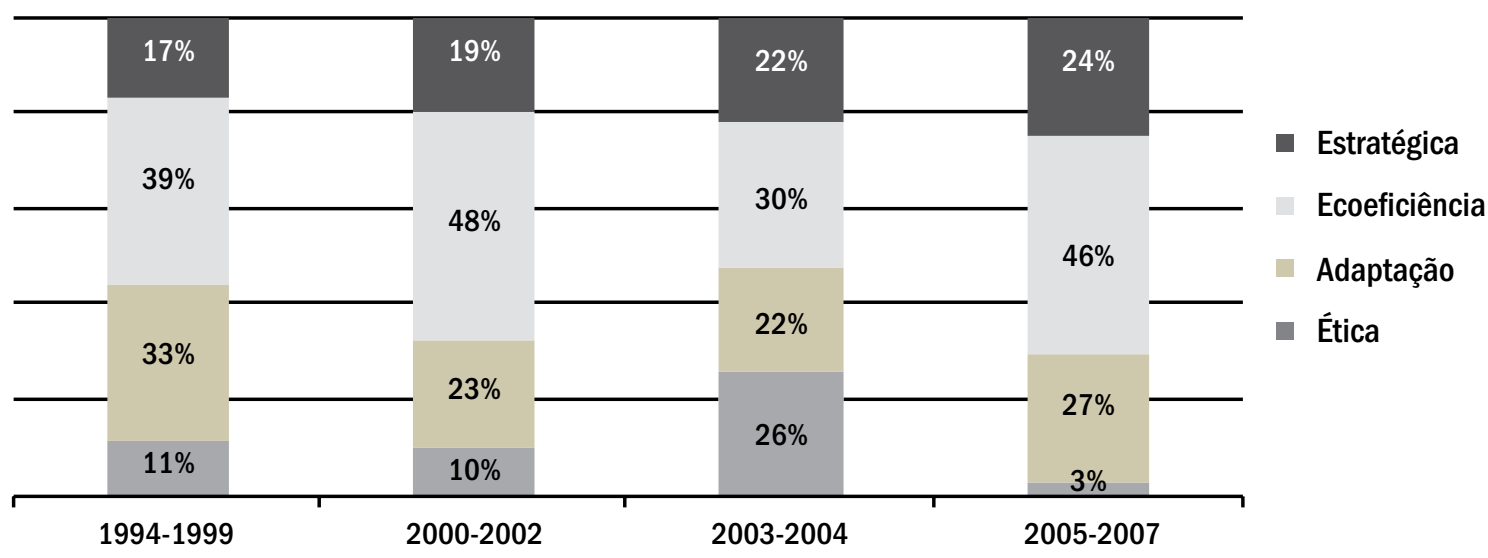


variação, contrariando uma expectativa de migração para a abordagem estratégica, seguindo a tendência da abordagem teórica do tema (ver seções 1 e 2).

A continuidade da pesquisa se deu pela leitura completa de todos os 97 estudos, buscando-se qualificá-los quanto aos objetivos propostos nas análises, a abrangência das iniciativas pesquisadas e a forma observada de envolvimento dos stakeholders. Novamente é proposta uma categorização (Quadro 2) baseada na revisão de literatura de estratégia e gestão de cadeias. Sua elaboração seguiu a evolução do pensamento estratégico mais recente, que dá ênfase a aspectos de cooperação na busca de parcerias para inovação e no desenvolvimento de ativos específicos.

$\mathrm{Na}$ análise da segunda categorização, os artigos que não corresponderam a nenhum dos enquadramentos es- tabelecidos foram classificados como zero (0), restando 96 artigos para análise em cada categoria.

Como já esperado, a verificação dos demais aspectos trouxe uma configuração não uniforme, $52 \%$ dos artigos não são categorizados consistentemente na mesma linha. Esse fato chama a atenção para a interseção dos movimentos na área de gestão de cadeias, isto é, há ainda mudança provocada pelas pressões regulatórias de competição, ao mesmo tempo que algumas iniciativas buscam ecodesing e capacidades dinâmicas nas interações.

Os objetivos declarados pelos estudos vêm crescentemente incluindo as temáticas de melhoria de desempenho e vantagem competitiva, no entanto a forma de envolvimento dos stakeholders é ainda muito baseada apenas na pressão ambiental (Gráfico 3).

\section{Quadro 2 - Categorização para análise dos artigos}

\begin{tabular}{|l|l|l|l|}
$\begin{array}{c}\text { FORMA DE } \\
\text { APRESENTAÇÃO }\end{array}$ & \multicolumn{1}{|c|}{$\begin{array}{c}\text { OBJEIIVO DA GESTÃO DA } \\
\text { CADEIA DE SUPRIMENTOS }\end{array}$} & $\begin{array}{c}\text { ABRANGÊNCIA DAS } \\
\text { INICIATIVAS TOMADAS }\end{array}$ & \multicolumn{1}{c|}{$\begin{array}{c}\text { FORMA DE ENVOLVIMENTO DOS } \\
\text { STAKEHOLDERS }\end{array}$} \\
\hline 1 - Adaptação & $\begin{array}{l}\text { 1- Evitar penalidades e a perda } \\
\text { de clientes e conseguir acesso a } \\
\text { mercado. }\end{array}$ & $\begin{array}{l}\text { 1- Atitudes isoladas, busca de } \\
\text { conformidade com padrão e } \\
\text { isomorfismo. }\end{array}$ & $\begin{array}{l}\text { 1- Pela pressão do mercado: de- } \\
\text { manda e competidores; pressões de } \\
\text { órgãos reguladores ou multilaterais } \\
\text { e pressão da sociedade civil. }\end{array}$ \\
\hline 2- Ecoeficiência & $\begin{array}{l}\text { 2- Melhorar desempenho eco- } \\
\text { nômico e ou ambiental, diminuir } \\
\text { impactos e gestão da cadeia. }\end{array}$ & $\begin{array}{l}\text { 2- Maior envolvimento dos parcei- } \\
\text { ros, mantendo foco nos interesses } \\
\text { da empresa e custos de transação. }\end{array}$ & $\begin{array}{l}\text { 2 - Controle, coordenação da ca- } \\
\text { deia, treinamento e seleção de } \\
\text { parceiros. }\end{array}$ \\
\hline 3- Estratégica & $\begin{array}{l}\text { 3- Inovação e vantagem compe- } \\
\text { titiva, formação de parcerias. }\end{array}$ & $\begin{array}{l}\text { 3- Desenvolvimento e cooperação } \\
\text { com demais atores e influência na } \\
\text { cadeia. }\end{array}$ & $\begin{array}{l}\text { 3 - Cooperação e desenvolvimento } \\
\text { de ativos específicos. }\end{array}$ \\
\hline
\end{tabular}

Gráfico 2 - Análise dos artigos: número de artigos por categoria

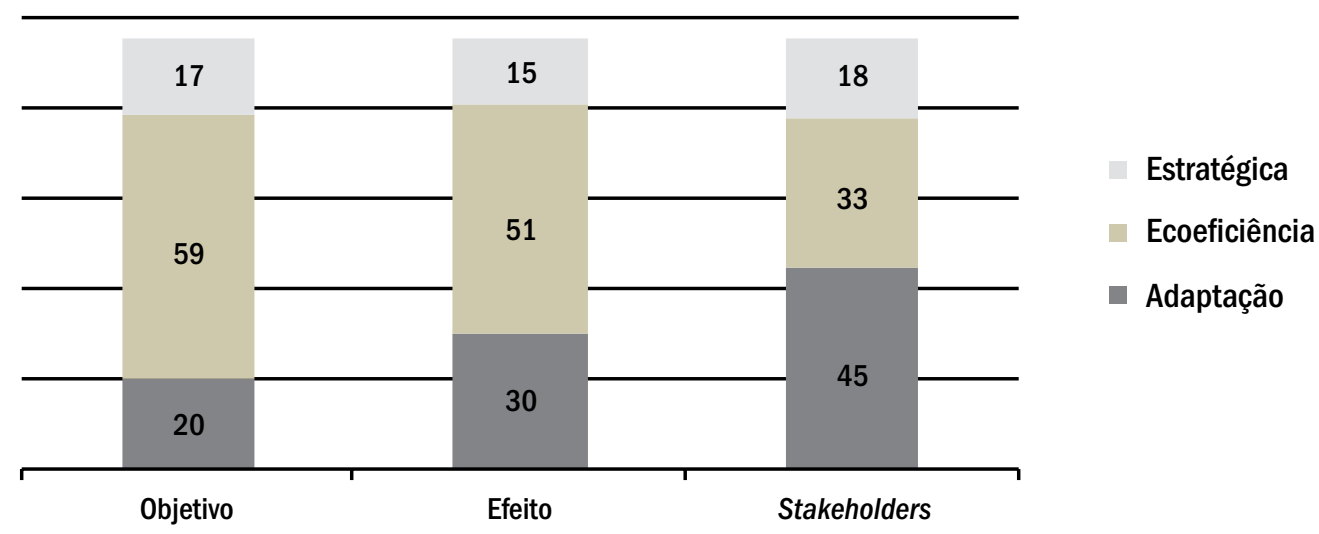


Por fim, a crescente incidência do uso do termo "sustentabilidade", identificado como palavra-chave (SEURING e MÜLLER, 2008), está correlacionada positivamente com o objetivo estratégico na gestão $(r=0,342 ; p=0)$ e com a colaboração com stakeholders $(r=0,306 ; p=0,001)$. Porém, a baixa correlação das variáveis é mais um indício da coexistência de objetivos e práticas desalinhados.

\section{CONSIDERAÇÕES FINAIS}

A pesquisa aqui elaborada difere daquela anteriormente feita por Seuring e Müller (2008) tanto pelo enfoque na abordagem estratégica da cadeia de suprimentos como pelo interesse em encontrar, nos achados empíricos, suporte às teorias de vantagem competitiva na gestão da cadeia. Esta última questão é especialmente relevante, pois na revisão dos artigos percebe-se que a abordagem teórica enfoca aspectos estratégicos que não são verificados nas análises empíricas.

A geração de valor nas relações organizacionais depende de aspectos de colaboração entre as partes, para além das relações de controle, extrapolando para a criação de ativos específicos que, pela complexidade social, não são facilmente imitáveis. Por outro lado, o comportamento colaborativo exige relações de confiança que superem o receio do oportunismo e a imposição de proteções processuais. Clássicos exemplos são dados pelo estudo do relacionamento das montadoras japonesas e seus fornecedores na criação de ativos específicos e cooperação (DYER, 1996).

\section{Gráfico 3 - Categorização ao longo do tempo}

Objetivo da gestão de cadeia de suprimentos:

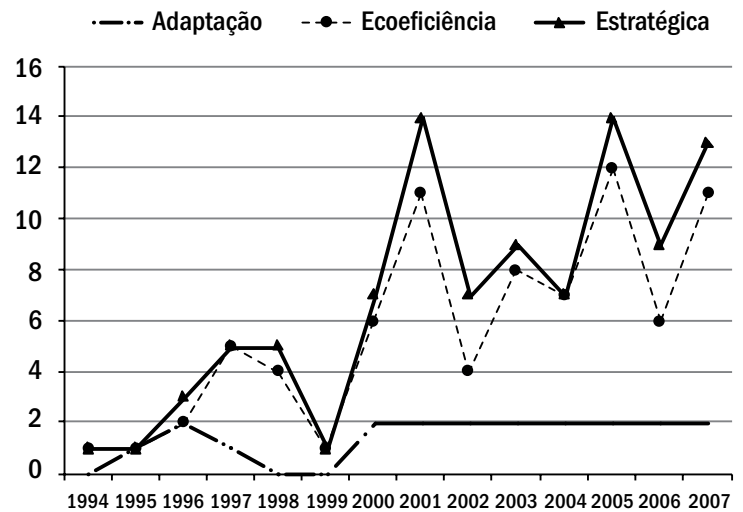

Os estudos de relações entre organizações como fonte de geração de valor não se restringem à temática da sustentabilidade. Argumentou-se, porém, que a empresa que expande seus relacionamentos na interação com stakeholders poderia gerar capacidades organizacionais e soluções proativas (BUYSSE e VERBEKE, 2003). O alinhamento estratégico e a geração de vantagem competitiva são vislumbrados na literatura de cadeia de suprimento verde e cadeia de suprimento sustentável. Portanto, a revisão empírica seguiu buscando as evidências.

Os resultados da análise dos artigos mostram que a relação entre parceiros comerciais é marcada pela definição de padrões mínimos, por procedimentos de controle e pela exclusão de fornecedores, em oposição a formas cooperativas e desenvolvimento conjunto. Os dados demonstram que a pressão externa (regulatória, do mercado e/ou da sociedade) é o enfoque preponderante na relação com os stakeholders (41\%), seguido pelos objetivos de melhoria de desempenho econômico e ambiental (30\%). Portanto, as iniciativas de Gestão Sustentável da Cadeia de Suprimentos pesquisadas encaixam-se no contexto da pressão ambiental por padrões mínimos e paridade competitiva. No mais, as relações são marcadas pelas dificuldades de colaboração e necessidade de gestão de custos de transação. Na constatação dessa lacuna, os próprios artigos pesquisados acabam por citar a necessidade de cooperação e aprendizagem conjunta.

O desafio na gestão de relacionamentos, para além dos custos de transação, extrapola o campo de interesse da sustentabilidade e se coloca para a gestão de cadeias como um todo; isto é, a necessidade de desenvolvimento

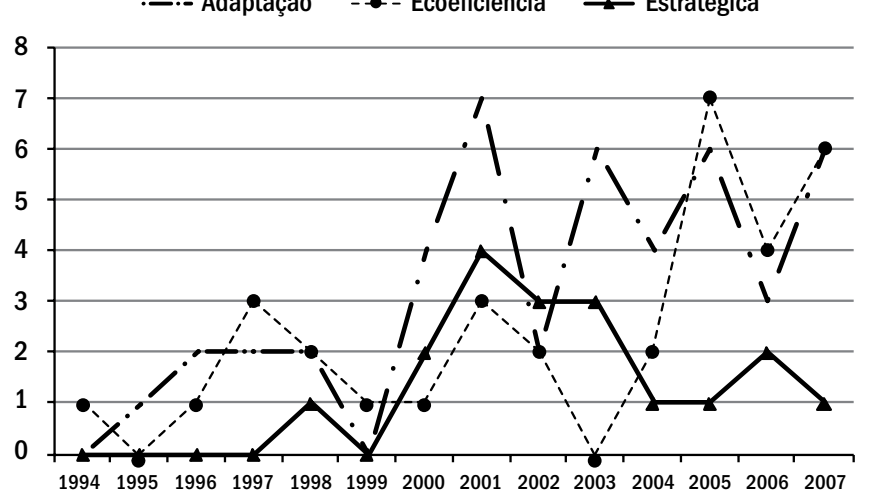


de relacionamentos colaborativos aplica-se de forma mais ampla. Particularmente na Gestão Sustentável da Cadeia de Suprimentos esse desafio se aplica na inovação em processos e design de produtos, que fundamentalmente depende da colaboração entre parceiros.

Entende-se que a necessidade de elaboração de novos estudos deveria endereçar as diferenças entre os campos teórico e prático. O aprofundamento na investigação das relações poderá trazer à luz as motivações das empresas ao expandir a relação com seus stakeholders e as dificuldades delas em desenvolver soluções conjuntas. Dessa forma, a Gestão Sustentável da Cadeia de Suprimentos alinha-se com as prioridades estratégicas da empresa, tanto na sua necessidade de legitimação como na busca de competitividade.

Por fim, a questão de desempenho econômico e ambiental está presente em grande parte dos estudos; no entanto, a origem da vantagem competitiva não está no desempenho, mas manifesta-se nele. Enquanto o desenvolvimento de recursos e capacidades for restrito, a manutenção do desempenho não pode ser considerada sustentada por uma vantagem competitiva.

\section{REFERÊNCIAS}

ANDERSEN, M; SKJOETT-LARSEN, T. Corporate social responsibility in global supply chains. Supply Chain Management: An International Journal, v. 14, n. 2, p. 75-86, 2009.

BAKKER, F. G. A; GROENEWEGEN, P; HOND, F. A bibliometric analysis of 30 years of research and the theory on corporate social responsibility and corporate social performance. Business and Society, v. 44, n. 3, p. 283-317, Sep, 2005.

BANSAL, P. Evolving sustainably: a longitudinal study of corporate sustainable development. Strategic Management Journal, v. 26, n. 3, p. 197218, 2005

BARNEY, J. B. Firm resources and sustained competitive advantage. Journal of Management, v. 17, n. 1, p. 99-120, 1991.

BARROS, A. J. P; LEHFELD, N. A. S. Fundamentos de metodologia. Um guia para a iniciação científica. São Paulo: McGraw-Hill, 1986.

BUYSSE, K; VERBEKE, A. Proactive environmental strategies: A stakeholder management perspective. Strategic Management Journal, v. 24, p. 453-470, 2003.

CARTER, C. R; JENNINGS, M. M. Logistics social responsibility: an integrative framework. Journal of Business Logistics, v. 23, n. 1, p. 145-180, 2002.
CARTER, C. R; ROGERS, D. S. A framework of sustainable supply chain management: moving toward new theory. International Hournal of Physical Distribution \& Logistics management, v. 38, n. 5, p. 360-387, 2008.

CERVO, A. L; BERVIAN, P. A. Metodologia cientifica: para uso de estudantes universitários. São Paulo: McGraw-Hill, 1983.

CHRISTMANN, P. Effects of 'best practices' of environmental management on cost advantage: the role of complementary assets. Academy of Management Journal, v. 43, n. 4, p. 663-680, 2000

COCHRAN, P; WOOD, R. A. Corporate social responsibility and financial performance. Academy of Management Journal, v. 27, n. 1, 1984

DARNALL, N; EDWARDS JR, D. Predicting the cost of environmental management system adoption: the role of capabilities, resources and ownership structure. Strategic Management Journal, v. 27, n. 4, p. 301-320, 2006.

DIERICKX, I; COOL, K. Asset stock accumulation and sustainability of competitive advantage. Management Science, v. 35, n. 12, p. 1504-1511, 1989 .

DIMAGGIO, P. J; POWELL, W. W. The iron cage revisited: institutional isomorphism and collective rationality in organizational fields. American Sociological Review, v.48, p.147-60, 1983

DYER, J. H. Specialized supplier networks as a source of competitive advantage: evidence from the auto industry. Strategic Management Journal, v. 17, n. 4, p. 271-291, 1996.

DYER, J. H; HATCH, N. W. Relation-specific capabilities and barriers to knowledge transfers: creating advantage through network relationships. Strategic Management Journal, v. 27, n. 8, p. 701-719, 2006.

DYER, J. H; SINGH, H. Relational view: cooperative strategy and sources of interorganizacional competitive advantage. Academy of Management Review, v. 23, n. 4, p. 660-679, 1998.

EESLEY, C; LENOX, M. J, Firm responses to secondary stakeholder action. Strategic Management Journal, v. 27, n. 8, p. 765-781, 2006.

FABER, N; JORNA, R; ENGELEN, J. The sustainability of sustainability - a study into conceptual foundations of the notion of sustainability. Journal of Environmental Assessment Policy and Management, v. 7, n. 1, Mar. 2005

GARRIGA, E; MELÉ, D. Corporate social responsibility theories: mapping the territory. Journal of Bussines Ethics, v. 53, 2004.

GERDE, V. W; WOKUTCH, R. E. 25 years and going strong: a content analysis of the first 25 years of the social issues in management division proceedings. Business \& Society, v. 37, n. 4, p. 414-446, 1998.

GOODLAND, R. The concept of environmental sustainability. Annual Review of Ecology and Systematics, v. 26, p. 1-24, 1995. 
HART, S. L. The natural-resource-based-view of the firm. Academy of Management Review, v. 20, p. 986-1014, 1995.

HEDGES, L. V. Meta-analysis. Journal of Educational Statistics, v. 17, n. 4, 1992.

KLEINDORFER, P. R; SINGHAL, K; VAN WASSENHOVE, L. N. Sustainable Operations Management. Production and Operations Management, v. 14, n. 4, p. 482-492, 2005.

LINTON, J; KLASSEN, R; JAYARAMAN, V. Sustainable supply chains: an introduction. Journal of Operations Management, v. 25, p. 1075-1082, 2007.

LÉLÉ, S. Sustainable development: a critical review. World Development, v. 19, n. $6,1991$.

MARKLEY, M. J; DAVIS, L. Exploring future competitive advantage through sustainable supply chains. International Journal of Physical Distribution E Logistics Management, v. 37, n. 9, p. 763-774, 2007.

MARREWIJK, M. Concepts and definitions of CSR and corporate sustainability: between agency and communion. Journal of Business Ethics, v. 44, 2003.

MARREWIJK, M; WERRE, M. Multiple levels of corporate sustainability. Journal of Business Ethics, v. 44, 2003.

MCGUIRE, J. B; SUNDGREN, A; SCHNEEWEIS, T. Corporate social responsibility and firm financial performance. Academy of Management Journal, v. 31, n. 4, p. 854-872, Dec. 1988.

ORSATO, R. J. Competitive environmental strategies: when does it pay to be green? California Management Review, v. 48, n. 2, p. 127-143, 2006.

PATTON, M. Q. Qualitative Research \& Education Methods. California: Sage Publications, 2002.

PORTER, M. Competitive Strategy: Techniques for Analyzing Industries and Competitors. New York: The Free Press, 1980

PORTER, M. E; VAN DER LINDE, C. Green and competitive: ending the stalemate. Harvard Business Review, v. 73, n. 5, p. 120-134, 1995.

PRESTON, L. E; O'BANNON, D. P. The corporate social-financial performance relationship. Business \& Society, v. 31, n. 4, p. 419-429, Dec. 1997.

RAO, P; HOLT, D. Do green supply chains lead to competitiveness and economic performance? International Journal of Operations \& Productions Management, v. 25, n.9, p. 898-916, 2005.

ROWLEY, T; BERMAN, S. A brand new brand of corporate social performance. Business \& Society, v. 39, n. 4, p. 397-418, 2000.
RUSSO, M. V; FOUTS, P. A. A resource-based perspective on corporate environmental performance and profitability. Academy of Management Journal, v. 40, n. 3, p. 534-559, 1997.

SAMPAIO, M. Diferentes interpretações do conceito de supply chain management. Revista Global, fev, 2007.

SEURING, S; MÜLLER, M. From a literature review to a conceptual framework for sustainable supply chain management. Journal of Cleaner Production, v. 16, p. 1699-1710, 2008.

SHARMA, S; VREDENBURG, H. Proactive corporate environmental strategy and the development of competitively valuable organizational capabilities. Strategic Management Journal, v. 19, n. 8, p. 729-753, 1998.

SHARMA, S; HENRIQUES, I. Stakeholder influences on sustainability practices in Canadian forest products industry. Strategic Management Journal, v. 26, n. 2, p. 159-180, Feb, 2005

SHRIVASTAVA, P. Ecocentric management for a risk society. Academy of Management Review, v. 20, n. 1, p. 118-137, 1995a.

SHRIVASTAVA, P. Environmental technologies and competitive advantage. Strategic Management Journal, v. 16, p. 183-200, 1995b.

SRIVASTAVA, S. Grenn suppy-chain management: a state-of-the-art literature review. International Journal of Management Reviews, v. 9, n. 1, p. 53-80, Mar, 2007.

SVENSSON, G. Aspects of sustainable supply chain management (SSCM) conceptual framework and empirical example. Supply Chain Management: An international Journal, v. 12, n. 4, p. 262-266, 2007.

TEECE, D. J. Explicating dynamic capabilities: the nature and microfoundations of (sustainable) enterprise performance. Strategic Management Journal, v. 28, n. 13, p. 1319-1350, 2007.

TEECE, D. J; PISANO, G. P; SHUEN, A. Dynamic capabilities and strategic management. Strategic Management Journal, v. 18, n. 7, p. 509-533, 1997.

TONELLI, M. J; CALDAS, M. P; LACOMBE, B. M. B; TINOCO, T. Produção acadêmica em recursos humanos no Brasil: 1991-2000. RAE-revista de administração de empresas, v. 43, n. 1, p. 107-122, 2003.

VAN NIMWEGEN, G; BOLLEN, L; HASSINK, H; THIJSSENES, T. A stakeholder perspective on mission statements: an international empirical study. International Journal of Organizational Analysis, v. 16, n. 1/2, p. 61-82, 2008.

VENKATRAMAN, N; SUBRAMANIAM, M. Theorizing the future of strategy: questions for shaping strategy research in the knowledge economy. In PETTIGREW, A; Thomas, H. e; Whittington, R. (Eds) Handbook of Strategy and Management. London: Sage, 2002. 
VERSCHOOR, C. C. A study of the link between a corporation's financial performance and its commitment to ethics. Journal of Business Ethics, v. 17, n. 13, p. 1509-1516, 1998.

WESTLEY, F; VREDENBURG, H. Sustainability and the corporation: criteria for aligning economic practice with environmental protection. Journal of Management Inquiry, v. 5, p. 104-119, 1996.

WILLIAMSON, O. E. Markets and Hierarchie. New York: Free Press, 1975.

\section{BASE DE 109 ARTIGOS PARA METAANÁLISE}

APAIAH, R. K; HENDRIX, E. M. T; MEERDINK, G; LINNEMANN, A. R. Qualitative methodology for efficient food chain design. Trends in Food Science \& Technology, v. 16, n. 5, 2005.

ARONSSON, H; BRODIN, M. H. The environmental impact of changing logistics structures. The International Journal of Logistics Management, v. 17, n. 3, 2006

BERGSTRO, M. K; SOLE, R. C; SHANAHAN, H. Professional food purchasers' practice in using environmental information. British Food Journal, v. 107, n. $5,2005$.

BLOWFIELD, M. Ethical supply chains in the cocoa, coffee and tea industries. Greener Management International, n. 43, 2003.

BOONS, F. Eco-design and integrated chain management: dealing with networks of stakeholders. The Journal of Sustainable Product Design, v. 5, 1998.

BOWEN, F. E; COUSINS, P. D; LAMMING, R. C; FARUK, A. C. Horses for courses: explaining the gap between the theory and practice of green supply. Greener Management International, n. 35, 2001

BOWEN, F. E; COUSINS, P. D; LAMMING, R. C; FARUK, A. C. The role of supply management capabilities in green supply. Production and Operations Management, v. 10, n. 2, 2001.

CANNING, L; HANMER-LLOYD, S. Managing the environmental adaptation process in supplier-customer relationships. Business Strategy and the Environment, v. 10, n. 4, 2001.

CARTER, C. R; JENNINGS, M. M. Logistics social responsibility - an integrative framework. Journal of Business Logistics, v. 23, n. 1, 2002.

CARTER, C. R. Ethical issues in international buyer-supplier relationships: a dyadic examination. Journal of Operations Management, v. 18, n. $2,2000$.

CARTER, C. R. Precursors of unethical behavior in global supplier management. The Journal of Supply Chain Management, v. 36, n. 1, 2000.
CARTER, C. R. Purchasing and social responsibility: a replication and extension. The Journal of Supply Chain Management, v. 40, n. 4, 2004.

CARTER, C. R. Purchasing social responsibility and firm performance: the keymediating roles of organizational learning and supplier performance. International Journal of Physical Distribution \& Logistics Management, v. 35, n. 3, 2005.

CARTER, C. R; JENNINGS, M. M. Social responsibility and supply chain relationships. Transportation Research Part E: Logistics and Transportation Review, v. 38, n. 1, 2002.

CARTER, C. R; JENNINGS, M. M. The role of purchasing in corporate social responsibility: a structural equation analysis. Journal of Business Logistics, v. 25, n. 1, 2004.

CARTER, C. R; KALE, R; GRIMM, C. M. Environmental purchasing and firm performance: an empirical investigation. Transportation Research Part E: Logistics and Transportation Review, v. 36, n. 3, 2000.

CARTER, C. R; CARTER, JR. Interorganizational determinants of environmental purchasing: initial evidence from the consumer products industries. Decision Sciences Journal, v. 29, n. 3, 1998.

CHOUINARD, Y; BROWN, M. S. Going organic - converting Patagonia's cotton product line. Journal of Industrial Ecology, v. 1, n. 1, 1997.

COOPER; R.W; FRANK, G. L; KEMP, R. A. A multinational comparison of key ethical issues, helps and challenges in the purchasing and supply management profession: the key implications for business and the professions. Journal of Business Ethics, v. 23, n. 1, 2000.

COOPER; R. W; FRANK, G. L; KEMP, R. A. Ethical issues, helps and challenges: perceptions of members of The Chartered Institute of Purchasing and Supply. European Journal of Purchasing \& Supply Management, v. 3. n. 4, 1997.

CORBETT, C. J; KIRSCH, D. A. International diffusion of ISO 14000 certification. Production and Operations Management, v. 10, n. 3, 2001.

COURVILLE, S. Use of indicators to compare supply chains in the coffee industry. Greener Management International, n. 43, 2003.

CRAMER, J. Responsiveness of industry to eco-efficiency improvements in the product chain: the case of Akzo Nobel. Business Strategy and the Environment, v. 9, n. 1, 2000.

CRAMER, J. M; VAN LENDERS, C. The process of chain-oriented environmental improvement at Van Hecke Catering. Greener Management International, n. 3, 2000

DANSE, M; WOLTERS, T. Sustainable coffee in the mainstream: the case of the SUSCOF consortium in Costa Rica. Greener Management International, n. $43,2003$. 
DAVIES, I. A; CRANE, A. Ethical decision making in fair trade companies. Journal of Business Ethics, v. 45, n. 1-2, 2003.

DE GROENE, A; HERMANS, M. Economic and other implications of integrated chain management: a case study. Journal of Cleaner Production, v. 6, n. 3-4, 1998.

DINIZ, J. D. A. S; FABBE-COSTES, N. Supply chain management and supply chain orientation: key factors for sustainable development projects in developing countries? International Journal of Logistics: Research and Applications, v. 10, n. 3, 2007.

DOBILAS, G; MACPHERSON, A. Environmental regulation and international sourcing policies of multinational firms. Growth and Change, v. 28, n. 1, 1997.

ELWOOD, H; CASE, S. Private sector pioneers: how companies are incorporating environmentally preferable purchasing. Greener Management International, n. 29, 2000

FORMAN, M; JORGENSEN, M. S. Organizing environmental supply chain management - experience from a sector with frequent product shifts and complex product chains: the case of the Danish textile sector. Greener Management International, n. 45, 2004.

FOSSGARD-MOSER, T. Promoting sustainable development through the enhancement of local employment and supply chain opportunities generated by energy companies: the case of the Shell group. Greener Management International, n. 43, 2003.

FREEMAN, D. Homeworkers in global supply chains. Greener Management International, n. 43, 2003.

GEFFEN, C. A; ROTHENBERG, S. Suppliers and environmental innovation: the automotive paint process. International Journal of Operations $\&$ Production Management, v. 20, n. 2, 2000.

GOLDBACH, M; SEURING, S; BACK, S. Coordinating sustainable cotton chains for the mass market - the case of the German mail order business OTTO. Greener Management International, n. 43, 2003.

GRAAFLAND, J. J. Sourcing ethics in the textile sector: the case of $C \& A$. Business Ethics: A European Review, v. 11, n. 3, 2002.

GRANKVIST, G; BIEL, A. The impact of environmental information on professional purchasers' choice of products. Business Strategy and the Environment, v. 16, n. 6, 2007.

GREEN, K; MORTEN, B; NEW, S. Purchasing and environmental management: Interactions, policies and opportunities. Business Strategy and the Environment, v. 5, n. 3, 1996.

GREEN, K; MORTEN, B; NEW, S. Green purchasing and supply policies: do they improve companies' environmental performance? Supply Chain Management: An International Journal, v. 3, n. 2, 1998.
HAGELAAR, G. J. L. F; VAN DER VORST, J. G. A. J. Environmental supply chain management: using life cycle assessment to structure supply chains. International Food and Agribusiness Review, v. 4, n. 4, 2002.

HALL, J. Environmental supply chain dynamics. Journal of Cleaner Production, v. 8, n. 6, 2000.

HALL, J. Environmental supply-chain innovation. Greener Management International, n. 35, 2001.

HAMPRECHT, J; CORSTEN, C; NOLL, M; MEIER, E. Controlling the sustainability of food supply chains. Supply Chain Management: An International Journal, v. 10, n. 1, 2005.

HANDFIELD, R; SROUFE, R; WALTON, S. Integrating environmental management and supply chain strategies. Business Strategy and the Environment, v. 14, n. 1, 2005.

HANDFIELD, R. B; WALTON, S. V; SEEGERS, L. K; MELNYK, S. A 'Green' value chain practices in the furniture industry. Journal of Operations Management, v.15, n. 4, 1997.

HOLT, D. Managing the interface between suppliers and organizations for environmental responsibility - an exploration of current practices in the UK. Corporate Social Responsibility and Environmental Management, v. 1, n. 2, 2004.

ILBERY, B; MAYE, D. Food supply chains and sustainability: evidence from specialist food producers in the Scottish English borders. Land Use Policy, v. 22, n. 4, 2005.

IRLAND, L. C. Developing markets for certified wood products: Greening the supply chain for construction materials. Journal of Industrial Ecology, v. 11, n. 1, 2007

JONES, A. An environmental assessment of food supply chains: A case study on dessert apples. Environmental Management, v. 30, n. 4, 2002.

JORGENSEN, A. L; KNUDSEN, J. S. Sustainable competitiveness in global value chains: how do small Danish firms behave? Corporate Governance, v. 6, n. 4,2006

KASSINIS, G. I; SOTERIOU, A. C. Greening the service profit chain: the impact of environmental management practices. Production and Operations Management, v. 12, n. 3, 2003

KING, A. A; LENOX, M. J. Lean and green? An empirical examination of the relationship between lean production and environmental performance. Production and Operations Management, v. 10, n. 3, 2001.

KLASSEN, R. D; VACHON, S. Collaboration and evaluation in the supply chain: The impact on plant-level environmental investment. Production and Operations Management, v. 12, n. 3, 2003.

KOCABASOGLU, C; PRAHINSKI, C; KLASSEN, R. D. Linking forward and reverse supply chain investments: the role of business uncertainty. Journal of Operations Management, v. 25, n. 6, 2007. 
KOGG, B. Greening a cotton-textile supply chain: a case study of the transition towards organic production without a powerful focal company. Greener Management International, n. 43, 2003.

KOPLIN, J; SEURING, S; MESTERHARM, M. Incorporating sustainability into supply management in the automotive industry: the case of the Volkswagen AG. Journal of Cleaner Production, v. 15, n. 11-12, 2007.

LAMMING, R. C; HAMPSON, J. P. The environment as a supply chain management issue. British Journal of Management, v. 7, 1996.

MAMIC, I. Managing global supply chain: the sports footwear, apparel and retail sectors. Journal of Business Ethics, v. 59, n. 1, 2005.

MATOS, S; HALL, J. Integrating sustainable development in the supply chain: the case of life cycle assessment in oil and gas and agricultural biotechnology. Journal of Operations Management, v. 25, n. 6, 2007.

MICHELSEN, O. Investigation of relationships in a supply chain in order to improve environmental performance. Cleaner Technology and Environmental Policy, v. 9, n. 2, 2007.

MICHELSEN, O; FET, A. M; DAHLSRUD, A. Eco-efficiency in extended supply chains: a case study of furniture production. Journal of Environmental Management, v. 79, n. 3, 2005.

MIN, H; GALLE, W. P. Green purchasing strategies: trends and implications. The Journal of Supply Chain Management, v. 33, n. 3, 1997.

MIN, H; GALLE, W. P Green purchasing practices of US firms. International Journal of Operations \& Production Management, v. 21, n. 9, 2001.

MINTCHEVA, V. Indicators for environmental policy integration in the food supply chain (the case of the tomato ketchup supply chain and the integrated product policy). Journal of Cleaner Production, v. 13, n. 7, 2005.

MURPHY, P. R; POIST, R. F. Socially responsible logistics: an exploratory study. Transportation Journal, v. 41, n. 4, 2002.

MURPHY, P. R; POIST, R. F. Green perspectives and practices: A "comparative logistics" study. Supply Chain Management: An International Journal, v. 8, n. 2, 2003.

MURPHY, P. R; POIST, R. F; BRAUNSCHWEIG, C. D. Management of environmental issues in logistics: current status and future potential. Transportation Journal, v. 34, n. 1, 1994.

MURPHY, P. R; POIST, R. F; BRAUNSCHWEIG, C. D. Role and relevance of logistics to corporate environmentalism - an empirical assessment. International Journal of Physical Distribution \& Logistics Management, v. 25, n. 2, 1995.

MURPHY, P. R; POIST, R. F; BRAUNSCHWEIG, C. D. Green logistics: comparative views of environmental progressives, moderates and conservatives. Journal of Business Logistics, v. 17, n. 1, 1996.
NARAYANASWAMY, V; SCOTT, J. A; NESS, J. N; LOCHHEAD, M. Resource flow and product chain analysis as practical tools to promote cleaner production initiatives. Journal of Cleaner Production, v. 11, n. 4, 2003.

NEILSON, J; PRITCHARD, B. Green coffee? The contradictions of global sustainability initiatives from an Indian perspective. Development Policy Review, v. 25, n. 3, 2007

PARTIDARIO, P. J; VERGRAGT, P. J. Planning of strategic innovation aimed at environmental sustainability: actor-networks, scenario acceptance and backcasting analysis within a polymeric coating chain. Futures, v. 34, n. 9-10, 2002.

PESONEN, H-L. Environmental management of value chains. Greener Management International, n. 33, 2001.

PREUSS, L. In dirty chains? Purchasing and greener manufacturing. Journal of Business Ethics, v. 34, n. 3-4, 2001.

PREUSS, L. Rhetoric and reality of corporate greening: a view from the supply chain management function. Business Strategy and the Environment, v. 14, n. 2, 2005

RAO, P. Greening the supply chain: a new initiative in South East Asia. International Journal of Operations \& Production Management, v. 22 , n. 6, 2002.

RAO, P. The greening of suppliers in the South East Asian context. Journal of Cleaner Production, v. 13, n. 9, 2005.

RAO, P; HOLT, D. Do green supply chains lead to competitiveness and economic performance? International Journal of Operations \& Production Management, v. 25, n. 9, 2005.

ROBERTS, S. Supply chain specific? Understanding the patchy success of ethical sourcing initiatives. Journal of Business Ethics, v. 44, n. 2, 2003

SEURING, S. Green supply chain costing - joint cost management in the polyester linings supply chain. Greener Management International, n. 33, 2001.

SEURING, S. Integrated chain management and supply chain management - comparative analysis and illustrative cases. Journal of Cleaner Production v. 12, n. $8-10,2004$

SIMPSON, D; POWER, D. J; SAMSON, D. Greening the automotive supply chain: a relationship perspective. International Journal of Operations \& Production Management, v. 27, n. 1, 2007.

STAINER, L; GULLY, A; STAINER, A. The UK food supply chain - an ethical perspective. Business Ethics: A European Review, v. 7, n. 4, 1998.

STOUGHTON, M; VOTTA, T. Implementing service-based chemical procurement: lessons and results. Journal of Cleaner Production, v. 11, n. 8, 2003 
TEUSCHER, P; GRÜNINGER, B; FERDINAND, N. Risk Management in sustainable supply chain management (SSCM): lessons learnt from the case of GMO-free soybeans. Corporate Social Responsibility and Environmental Management, v. 13, n. 1, 2006.

THEYEL, G. Customer and supplier relations for environmental performance. Greener Management International, n. 35, 2001.

TROWBRIDGE, P. A case study of green supply-chain management at advanced micro devices. Greener Management International, n. 35, 2001.

VACHON, S. Green supply chain practices and the selection of environmental technologies. International Journal of Production Research, v. 45, n. 18,2007 .

VACHON, S; KLASSEN, R. D. Extending green practices across the supply chain. The impact of upstream and downstream integration. International Journal of Operations \& Production Management, v. 26, n. 7, 2006.

VACHON, S; KLASSEN, R. D Green project partnership in the supply chain: the case of the package printing industry. Journal of Cleaner Production, v. 14, n. 6-7, 2006.

VASILEIOU, K; MORRIS, J. The sustainability of the supply chain for fresh potatoes in Britain. Supply Chain Management: An International Journal, v. 11, n. 4,2006

VERGHESE, K; LEWIS, H. Environmental innovation in industrial packaging: a supply chain approach. International Journal of Production Research, v. 45, n. 18, 2007.

VERMEULEN, W. J. V; RAS P. J. The challenge of greening global product chains: meeting both ends. Sustainable Development, v. 14, n. 4, 2006

VERSCHOOR, A. H; REIJNDERS, L. How the purchasing department can contribute to toxics reduction. Journal of Cleaner Production, v. 5, n. 3, 1997.

VIDAL, N; KOZAK, R; COHEN, D. Chain of custody certification: an assessment of the North American solid wood sector. Forest Policy and Economics, v. 7, n. 3, 2005.

WARREN, J. P; RHODES, E; CARTER, R. A total product system concept - a case study of the Smart ${ }^{\mathrm{TM}}$ automobile. Greener Management International, n. 35, 2001.

WELFORD, R; FROST, S. Corporate social responsibility in Asian supply chains. Corporate Social Responsibility and Environmental Management, v. 13, n. 3, 2006
WINSTANLEY, D; CLARK, J; LEESON, H. Approaches to child labour in the supply chain. Business Ethics: A European Review, v. 11, n. 3, 2003.

WYCHERLY, I. M. Greening supply chains: the case of the Body Shop International. Business Strategy and the Environment, v. 8, n. 2, 1999.

ZHU, Q; COTE, R. P. Integrating green supply chain management into an embryonic eco-industrial development: a case study of the Guitang Group. Journal of Cleaner Production, v. 12, n. 8-10, 2004.

ZHU, Q; GENG, Y. Integrating environmental issues into supplier selection and management: a study of large and medium-sized state-owned enterprises in China. Greener Management International, n. 35, 2001.

ZHU, Q; SARKIS, J. Relationships between operational practices and performance among early adopters of green supply chain management practices in Chinese manufacturing enterprises. Journal of Operations Management, v. 22, n. 3, 2004

ZHU, Q; SARKIS, J. The link between quality management and environmental management in firms of differing size: an analysis of organizations in China. Environmental Quality Management, v. 13, n. 3, 2004

ZHU, Q; SARKIS, J. An inter-sectoral comparison of green supply chain management in China: drivers and practices. Journal of Cleaner Production v. 14, n. 5,2006

ZHU, Q; SARKIS, J. The moderating effects of institutional pressures on emergent green supply chain practices and performance. International Journal of Production Research, v. 45, n. 18, 2007.

ZHU, Q; SARKIS, J; GENG, Y. Green supply chain management in China: pressures, practices and performance. International Journal of Operations E Production Management, v. 25, n. 5, 2005

ZHU, Q; SARKIS, J; LAI, K. H. Green supply chain management: pressures, practices and performance within the Chinese automobile industry Journal of Cleaner Production, v. 15, n. 11, 2007.

ZHU, Q; SARKIS, J; LAI, K. H. Initiatives and outcomes of green supply chain management implementation by Chinese manufacturers. Journal of Environmental Management, v. 25, n. 6, 2007.

ZSIDISIN, G. A; HENDRICK, T. E. Purchasing's involvement in environmental issues: a multi-country perspective. Industrial Management $\&$ Data Systems, v. 98, n. 7-8, 1998 